\title{
Age-Specific Signaling Responses to Skeletal Muscle Disuse: A Literature Review
}

\author{
Toshinori Yoshihara* \\ Graduate School of Health and Sports Science, Juntendo University, Japan \\ *Corresponding author: Toshinori Yoshihara, Graduate School of Health and Sports Science, Juntendo University, 1-1 \\ Hirakagakuendai, Inzai, Chiba 270-1695, Japan
}

\section{ARTICLE INFO}

Received: 峵 March 07, 2019

Published: 豐 March 18, 2019

Citation: Toshinori Yoshihara. AgeSpecific Signaling Responses to Skeletal Muscle Disuse: A Literature Review. Biomed J Sci \& Tech Res 16(1)-2019. BJSTR. MS.ID.002797.

\section{ABSTRACT}

Aging is associated with progressive muscle wasting. However, whether aging exacerbates the atrophy that is induced by muscle disuse is so far unknown. Some studies found a higher rate of muscle atrophy in aged rodents ( $>22$ months old) than in young ones (3-10 months old), whereas other investigators reported that aged muscles have a similar or lesser rate of atrophy in response to hindlimb unloading-induced muscle disuse than muscles in younger rodents. There is growing evidence that the differential responses of genes and signaling to skeletal muscle disuse in rodents and humans are age-related. This literature review summarizes the age-specific different responses to disuse observed in skeletal muscle and describes the potential underlying mechanisms for these differences.

Keywords: Age-Specific Differences; Disuse Muscle Atrophy; Apoptosis; Growth Arrest; DNA Damage-Inducible $45 \alpha$

\section{Introduction}

Aging is associated with a progressive loss of skeletal muscle mass and strength. This age-related muscle wasting is called sarcopenia and related to frailty, a shortened health span, and impaired quality of life in older (e.g. > 65 years) individuals [1]. However, whether aging exacerbates the atrophy induced by muscle disuse is so far unknown. A few studies found a higher rate of muscle atrophy in aged ( $>22$ months old) rodents than in young (3-10 months old) rodents [2,3], whereas other investigators reported that aged muscles show a similar or lesser extent of atrophy in response to muscle disuse [4-9]. These data suggest that aging does not increase the rate of atrophy caused by muscle disuse. Notably, growing evidence indicates that the different responses to skeletal muscle disuse in rodents and humans are age-related [912]. This implies that there might be age-related differences in the mechanisms that induce disuse muscle atrophy, even if the rate at which this atrophy occurs is similar between age groups. Skeletal muscle wasting induced by disuse such as limb immobilization and hindlimb unloading involves multiple signaling pathways [13], but the exact molecular mechanisms are not completely understood.
Growing evidence implies a possibility that the differential response of genes and signaling transducers to skeletal muscle disuse in rodents and humans is related to age [9-12]. The goals of this literature review are to summarize age-specific differences in the response to muscle disuse and to discuss the underlying mechanisms responsible for the differences in disuse muscle atrophy depending on age. A better understanding of these mechanisms in aged skeletal muscle is necessary to minimize the negative effects of muscle disuse on elderly individuals.

\section{Age-Specific Differences in Signaling Pathways in Response to Muscle Disuse in Rodents}

Leeuwenburgh et al. [9] compared the apoptotic response in the soleus muscle after 14 days of hindlimb unloading between young (6 months old) and old (32 months old) male Fischer 344 $\times$ Brown Norway rats. They demonstrated that old rats showed higher levels of apoptosis [terminal deoxynucleotidyl transferasemediated dUTP nick-end labeling (TUNEL) and DNA fragmentation] in their soleus muscle in response to hindlimb unloading compared to young rats despite a similar extent of muscular atrophy in the 
two groups. Moreover, the proapoptotic endonuclease G (EndoG, a mitochondrion-specific nuclease) increased only in the old, but not in the young rats after 14 days of unloading. In contrast, while caspase- 3 activity tended to increase with age, the response to unloading was similar in both groups. Moreover, Marzetti et al. indicated that the mitochondrial caspase-independent apoptotic pathway such as EndoG or apoptosis-inducing factors may play a more prominent role in age-related skeletal muscle loss than caspase-mediated apoptosis [10]. These data suggest that the processes involved in apoptosis are distinct in the muscles of young and old rats, and that muscle loss is partly mediated by the subsarcolemmal mitochondria apoptotic pathway through EndoG translocation to the nucleus, but less so by caspase-mediated apoptosis.

Recent evidence suggests that growth arrest and DNA damageinducible $45 \alpha$ (Gadd $45 \alpha$ ) plays an important role in hindlimb unloading-induced muscle atrophy, especially in aged $>29$ months old) skeletal muscle [14]. Gadd $45 \alpha$ is primarily a myonuclear protein that is rapidly induced by DNA damage and various kinds of stress, resulting in cell cycle arrest and/or apoptosis, and also actively participates in DNA repair mechanisms. A previous study indicated that Gadd $45 \alpha$ alters skeletal muscle gene expression and stimulates protein breakdown, reduces protein synthesis, decreases mitochondria, inhibits anabolic signaling, and, ultimately, causes muscle fiber atrophy when induced by different muscle stresses such as fasting, denervation, and immobilization [15]. Indeed, Baehr et al. indicated that Gadd $45 \alpha$ mRNA expression was significantly increased in the tibialis anterior muscle of 29-monthold male Fischer $344 \times$ Brown Norway rats after 14 days of hindlimb unloading, but not in 9-month-old rats [14].

They also demonstrated that Gadd $45 \alpha$ mRNA expression was significantly increased after three and seven days of hindlimb unloading in the gastrocnemius muscle of 29-month-old male Fischer $344 \times$ Brown Norway rats, which further showed greater Gadd $45 \alpha$ mRNA expression during the entire unloading period compared with 9-month-old rats [11]. In summary, age-related differential Gadd $45 \alpha$ and EndoG signaling plays an important role in the skeletal muscle response to disuse in animal models. Additionally, Gadd $45 \alpha$ activation induces an age-related delay in recovery from atrophy [14]. Thus, based on these animal studies, it appears important to develop a potential therapeutic countermeasure prior to muscle disuse and identify target molecules to protect aged muscle against disuse muscle atrophy.

\section{Age-Specific Differences in Signaling Pathways in Response to Muscle Disuse in Humans}

Regarding human skeletal muscle, one study [12] has compared age-related mechanisms in disuse muscle atrophy between young (21-27 years) and old (60-72 years) adults. There were no age-specific differences in the upregulation of ubiquitin E3 ligase (Atrogin-1 and muscle RING-Finger protein-1) or the downregulation of peroxisome proliferator-activated receptor gamma coactivator-1 alpha gene expression. However, the data revealed an age-specific upregulation of $\mathrm{Bcl}-2$-associated $\mathrm{X}$ protein and tumor protein 53 in the muscle of old adults after two days of immobility with a significant increase in TUNEL positive nuclei. This was followed by further increasing levels after four days, when there was also an increase of these parameters in the muscle of young adults. The study further found a selective decrease in protein kinase B/Akt phosphorylation status and greater reduction in ribosomal protein S6 phosphorylation in young adults that may be associated with a greater decline in the muscle fiber cross-sectional area in young as compared to the one in old individuals $(-19.9 \%$ and $-12.6 \%$, respectively). Overall, information on age-specific differences in muscle adaptation to disuse in humans is still very limited. Thus, future studies are required to clarify the different mechanisms of disuse-induced muscular atrophy in human skeletal muscle related to age.

\section{Summary and Conclusion}

Although it remains unclear whether aging accelerates the rate of atrophy induced by muscle disuse, growing evidence indicates that there are age-related differences in the signaling response to muscle disuse in both humans and rodents. Investigating the mechanisms responsible for age-specific responses in disuse muscle atrophy may provide unique information that can help to identify biological targets for interventions aimed at developing therapeutic approaches to prevent disuse muscle atrophy in aged muscle.

\section{Acknowledgment}

This work was partly supported by grants from the Japan Society for the Promotion of Science (JSPS) KAKENHI Grant No. 17K01765 (T. Yoshihara). We would like to thank Editage (www. editage.jp) for English language editing.

\section{References}

1. Demontis F, Piccirillo R, Goldberg AL, Perrimon N (2013) Mechanisms of skeletal muscle aging: insights from Drosophila and mammalian models. Dis Model Mech 6(6): 1339-1352.

2. Deschenes, MR, Britt, AA, Chandler WC (2001) A comparison of the effects of unloading in young adult and aged skeletal muscle. Med Sci Sports Exerc 33(9): 1477-1483.

3. Deschenes MR, Sherman EG, Glass EK (2012) The effects of prehabilitative conditioning on unloading-induced adaptations in young and aged neuromuscular systems. Exp Gerontol 47(9): 687-694.

4. Siu PM, Pistilli EE, Alway SE (2005) Apoptotic responses to hindlimb suspension in gastrocnemius muscles from young adult and aged rats. Am J Physiol Regul Integr Comp Physiol 289(4): R1015-1026.

5. Brown M, Taylor J (2005) Prehabilitation and rehabilitation for attenuating hindlimb unweighting effects on skeletal muscle and gait in adult and old rats. Arch Phys Med Rehabil 86(12): 2261-2269.

6. Pattison JS, Folk LC, Madsen RW, Booth FW (2003) Selected Contribution: Identification of differentially expressed genes between young and old rat soleus muscle during recovery from immobilization-induced atrophy. J Appl Physiol (1985) 95(5): 2171-2179. 
7. Alway SE, Lowe DA, Chen KD (2001) The effects of age and hindlimb supension on the levels of expression of the myogenic regulatory factors MyoD and myogenin in rat fast and slow skeletal muscles. Exp Physiol 86(4): 509-517.

8. Hwee DT, Bodine SC (2009) Age-related deficit in load-induced skeletal muscle growth. J Gerontol A Biol Sci Med Sci 64(6): 618-628.

9. Leeuwenburgh C, Gurley CM, Strotman BA, Dupont Versteegden EE (2005) Age-related differences in apoptosis with disuse atrophy in soleus muscle. Am J Physiol Regul Integr Comp Physiol 288(5): R1288-R1296.

10. Marzetti E, Wohlgemuth SE, Lees HA, Chung HY, Giovannini S, et al (2008) Age-related activation of mitochondrial caspase-independent apoptotic signaling in rat gastrocnemius muscle. Mech Ageing Dev 129(9): 542-549.

11. Baehr LM, West DWD, Marshall AG, Marcotte GR, Baar K, et al. (2017) Muscle-specific and age-related changes in protein synthesis and

\section{ISSN: 2574-1241}

DOI: 10.26717/BJSTR.2019.16.002797

Toshinori Yoshihara. Biomed J Sci \& Tech Res

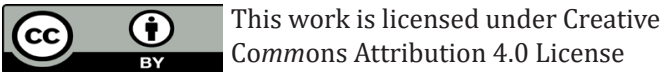

Submission Link: https://biomedres.us/submit-manuscript.php protein degradation in response to hindlimb unloading in rats. J Appl Physiol (1985) 122(5): 1336-1350.

12. Suetta C, Frandsen U, Jensen L, Jensen MM, Jespersen JG, et al. (2012) Aging affects the transcriptional regulation of human skeletal muscle disuse atrophy. PLoS ONE 7(12): e51238.

13. Gao Y, Arfat Y, Wang H, Goswami N (2018) Muscle Atrophy Induced by Mechanical Unloading: Mechanisms and Potential Countermeasures. Front Physiol 9: 235.

14. Baehr LM, West DW, Marcotte G, Marshall AG, De Sousa LG, et al. (2016) Age-related deficits in skeletal muscle recovery following disuse are associated with neuromuscular junction instability and ER stress, not impaired protein synthesis. Aging (Albany NY) 8(1): 127-146.

15. Ebert SM, Dyle MC, Kunkel SD, Bullard SA, Bongers KS, et al. (2012) Stressinduced skeletal muscle Gadd45a expression reprograms myonuclei and causes muscle atrophy. J Biol Chem 287(33): 27290-27301.

$\begin{array}{ll}\text { BIOMEDICAL } & \text { Assets of Publishing with us } \\ \text { RESEARCHES } & \text { - Global archiving of articles } \\ & \text { - Immediate, unrestricted online access } \\ \end{array}$

